\title{
Supramolecular Multilayered Templates for Fabricating Nanometer- Precise Spacings: Implications for the Next-Generation of Devices Integrating Nanogap/Nanochannel Components
}

\author{
Hadi Arjmandi-Tash, ${ }^{\dagger}$ Pauline M.G. van Deursen, ${ }^{\dagger}$ Amedeo Bellunato, ${ }^{\dagger}$ Clarisse de Sere, \\ Zhanna Overchenko, Karthick Babu Sai Sankar Gupta, and Grégory F. Schneider*
}

Cite This: ACS Appl. Nano Mater. 2020, 3, 10586-10590

Read Online

\section{ACCESS |}

Џlll Metrics \& More

Article Recommendations

Supporting Information

ABSTRACT: Molecular transistors, electromagnetic waveguides, plasmonic devices, and novel generations of nanofluidic channels comprise precisely separated gaps of nanometric and subnanometric spacing. Nonetheless, fabricating a nanogap/nanochannel is a technological challenge, currently tackled by several approaches such as breakdown electromigration and lithography. The aforementioned techniques, though, are limited, respectively, in terms of gap stability and ultimate resolution. Here, nanogaps/ nanochannels are templated via the microtomy of metallic thin films embedded in a polymer matrix and precisely separated by a nanometric, sacrificial layer of polyelectrolytes grown via the layerby-layer (LbL) approach. The versatility of the LbL technique,

\section{$\mathrm{Au}$}

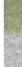

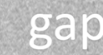

$\mathrm{Au}$ both in terms of the number of layers and composition of polyelectrolytes, allows to finely tune the spacing across the gap; the LbL template can further be removed by plasma etching. Our findings pave the path toward the realization of molecularly defined functional spacings at the nanometer-scale for the modular implementation of devices integrating nanogap/nanochannel components.

KEYWORDS: nanogap, nanochannel, layer-by-layer assembly, polyelectrolyte, microtomy, nanoskiving

\section{INTRODUCTION}

The fine control of the spacing across two parallel structures/ walls is critical for the design of nanogap/nanochannel devices $^{1,2}$ and their application into tunneling junctions, ${ }^{3-6}$ molecular transistors, ${ }^{5,7-10}$ waveguides, ${ }^{11-16}$ plasmonic devices, ${ }^{17,18}$ and state-of-the-art nanofluidic channels. ${ }^{19}$ For instance, the characteristics of devices such as molecular transistors rely on the specific molecular structure of the bridging element and most importantly require electrodes separated by a gap having a width comparable to that of a single molecule, ${ }^{20}$ that is, few nanometers. Similarly, tunneling junctions are based on nearly touching electrodes-typically also separated by a few nanometers-across which a tunneling current detects $^{21}$ or probes the electronic transport through single molecules. ${ }^{22-25}$ Nonetheless, the precise and controllable fabrication of so-close nanogaps/nanochannels hits the intrinsic limits of conventional optical and electron-beam lithography. ${ }^{26}$

Microtomy-the process of skiving nanometer-thick slabs out of a polymeric block which in our case embeds thin films-has been successfully employed in surface micromachining of complex nanostructures. ${ }^{27}$ Facile operation and affordable process costs are among the important advantages of the approach. The process does not require a cleanroom environment and is applicable for a wide range of materials (metals, ceramics, semiconductors, two-dimensional materials, polymers, etc.) and geometries. The functionality of the device to be made is controlled by the properties of the embedded thin films (number of layers, thickness of the films, and spacing between them). Highly complex structures can be made by overdepositing multiple slabs. ${ }^{19}$ Particularly sub-nanometer gaps between metallic nanostructures have been realized by using a graphene template between two metallic films, reaching the ultimate lower limit of physically achievable gap size. ${ }^{28}$ Larger gaps can potentially be achieved by increasing the number of graphene layers (overdepositing monolayers); in practice, the stress exerted on the structure during the skiving, however, may exceed the weak van der Waals interaction between the layers causing uncontrolled delamination. More-

Received: June 9, 2020

Accepted: September 3, 2020

Published: September 3, 2020 
over, scaling up the gap size (>few nanometers) by overdepositing numerous multilayers is impractical. Note that replacing multilayer graphene with an ultrathin sacrificial solidstate material (e.g., deposited films) would lack accuracy as a result of the inhomogeneous deposition typically observed when decreasing the thickness. ${ }^{29}$ While innovative strategies have come up in order to achieve precisely aligned nanogaps/ nanochannels, ${ }^{30-32}$ several drawbacks remain, mainly the stability of the spacing with temperature ${ }^{33,34}$ and the formation of process residuals detrimental for the performances of the eventual device. $^{35}$

In this article, we employ the layer-by-layer ( $\mathrm{LbL})$ deposition $^{36}$ to grow multilayered stacks of polyelectrolytes of precisely controlled thicknesses between two large area metallic thin films. The stacks are subsequently embedded into a polymeric block; skiving the surface of the block with microtomy achieves metallic nanorods spaced by a sacrificial layer of polyelectrolytes. ${ }^{27,37,38} \mathrm{Next}$, the molecular-length spacing is opened up using plasma reactive etching of the sacrificial layer. Compared to existing reports (e.g., using monolayer graphene templates), the gap size-controlled by the multilayered polyelectrolytes - can vary over a wide range (few nanometers to few tens of nanometers) with an accuracy down to nanometer scale. The strong electrostatic interaction between the layers of polyelectrolytes and the covalent bonds between the metal and adjacent layer minimize the risk of delamination during the process. The polymeric support permits the agile manipulation of such nanostructures, their precise alignment, and the assembly into a well-defined device architecture. As a remarkable example, we already demonstrated that overdepositing two consecutive slabs yields complex nanofluidic architectures. ${ }^{19}$

\section{EXPERIMENTAL SECTION}

In order to form nanogaps/nanochannels, a multilayered polyelectrolyte stack is stepwise deposited on a gold surface prefunctionalized with a mixed self-assembled monolayer (SAM) of amino- and alkylthiols grown from a solution of $5 \mathrm{mM}$ 1-dodecanethiol and $5 \mathrm{mM} 11$ amino-1-undecanethiol in ethanol ${ }^{39}$ (Figure 1a).

The aminothiols protonate in acidic solutions, conferring the gold surface positive charges and allowing the adsorption of the first layer of the negatively charged poly(sodium 4-styrenesulfonate) (PSS) via electrostatic interaction. ${ }^{36}$ The self-assembly was characterized by ellipsometry (Figure 1b). Uniform, ordered monolayers formed only from ethanol solutions with a concentration of aminothiols below $40 \%$ in volume (see the Supporting Information). Higher concentrations yielded thicker layers because of the formation of disordered thiol assemblies, which turned out to be unsuitable for the deposition of further polyelectrolyte layers. Figure 1c shows the X-ray photon electron spectroscopy (XPS) of a SAM obtained from a solution containing $30 \%$ of aminothiols in volume. In this XPS plot, the low-intensity N $1 S$ peak around $399 \mathrm{eV}$ rises from the emission of the nitrogen atoms of the amine group terminating each aminothiol. The low intensity suggests the formation of a uniform distribution of aminothiols across the SAM, promoting the homogeneous charging of the gold surface.

Following the adsorption of the first layer of the negatively charged PSS, the film of polyelectrolytes grows via alternate deposition of oppositely charged PAH and PSS, layer-by-layer. At last, a thiolated $\mathrm{PAH}$ needs to be deposited as a top layer to ultimately yield a strong adhesion to the second gold film constituting the second nanogap/ nanochannel walls (Figure 1d). Importantly, the partial thiolation of the last PAH layer ensures both the electrostatic interaction with the underlying PSS and the upper gold film via thiol bonding, therefore strengthening the mechanical stability of the interface. a)
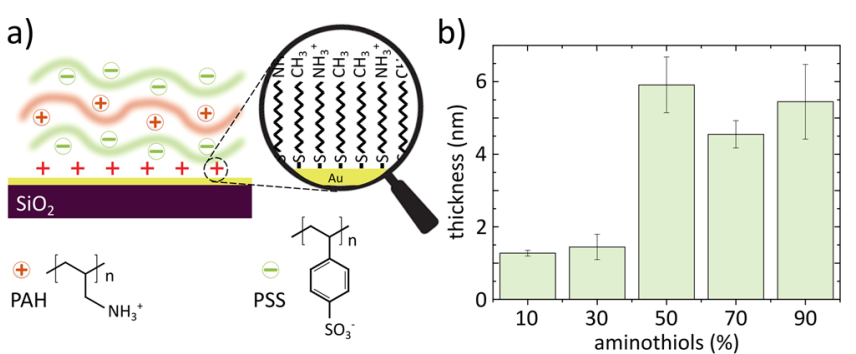

c)

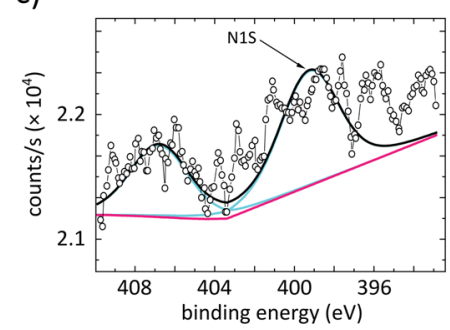

d)

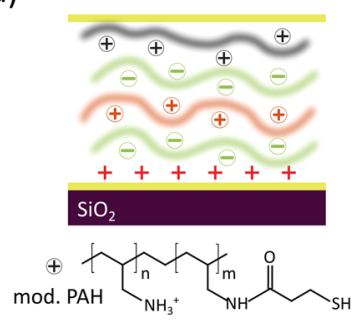

Figure 1. Alternated LbL deposition of polyelectrolytes to form a nanogap. (a) A supported gold film (yellow) on a $\mathrm{Si} / \mathrm{SiO}_{2}$ wafer is functionalized with a mixed SAM enriched with protonated aminothiols (side view). (b) Characterization of mixed SAM of 1dodecanethiol and 11-amino-1-undecanethiol by ellipsometry as a function of the amino-thiol concentration in volume. (c) X-ray photoelectron spectroscopy (XPS) of a mixed SAM with $30 \%$ in volume of 11-amino-1-undecanethiol with a fit of the $\mathrm{N}$ 1S peak belonging to the amines present in the SAM (solid line). (d) Illustration of the layered architecture: The upmost layer of polyelectrolytes (black) is the thiolated poly1-(allylamine hydrochloride) (PAH). Yellow layers correspond to the gold films.

The synthesis of the last thiolated poly(allyl-amine) layer is depicted in Figure 2a. The addition of a thiol functionality results from the reaction of a disulfide precursor with the dandling amines of $\mathrm{PAH}$, where (ii) is obtained from the esterification of (i) 3,3'dithiopropionic acid with $N$-hydroxysuccinimide. The reactive amines of the PAH open the pyrrolidone heterocycle of (ii) crosslinking the PAH via disulfide branches (iii). Afterward, dithiothreitol breaks the disulfide, forming thiol moieties. Figure $2 \mathrm{~b}$ shows the solidstate ${ }^{13} \mathrm{C}$ CPMAS nuclear magnetic resonance (NMR) characterization of the PAH before and after conversion (brown and black spectra, respectively). Thiol moieties are plugged to the $\mathrm{PAH}$ backbone via a carbonyl linker, followed by methyl spacers. The presence of sulfur and oxygen atoms in (ii) make the methylene carbon attached to the nitrogen of PAH peaks move from $32.98 \mathrm{ppm}$ to $36.78 \mathrm{ppm}$; this downfield shift of $4 \mathrm{ppm}$ clearly proves that the reaction has proceeded further. Additionally, the presence of the carbonyl linker is further confirmed by a major peak at $172.98 \mathrm{ppm}$ and a small peak at $160.89 \mathrm{ppm}$. This minor peak could be formed because of the presence of hydrogen bonding in the polymer, leading to more upfield shift.

When immersed in a $\mathrm{KCl}$ electrolyte, the residual amines of the thiolated PAH protonate, driving its deposition on top of the multilayered film of polyelectrolytes (Figure $2 \mathrm{c}$ ). Starting from a negative layer of PSS, the multilayered film grows linearly, stepwise at a pace down to $1.5 \mathrm{~nm}$ per layer. Remarkably, the modified PAH grows thicker than the layers underneath. The lower content of amines on its backbone yields increased thickness: less amines protonate in $\mathrm{KCl}$ solution, reducing the overall charging of the polyelectrolyte. Thus, the outmost PSS layer demands for a higher amount of modified PAH in order to screen its negative charge.

At last, the polyelectrolytes are coated with a second layer of gold, yielding two metallic thin films separated by a tunable, molecular spacer of polyelectrolytes. This stack is then lifted from its substrate (Figure 3a) and immersed within a photocurable polymer of pentaerythritol tetra(3-mercaptopropionate) and triallyl-1,3,5-tria- 
a)

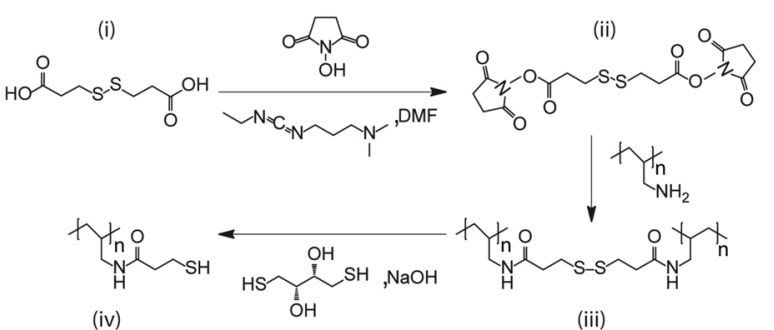

b)

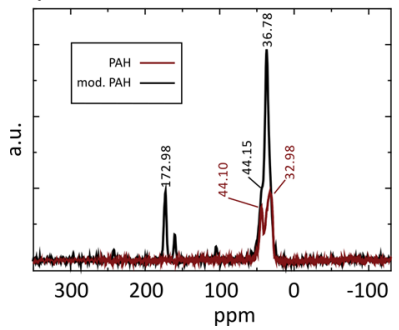

C)

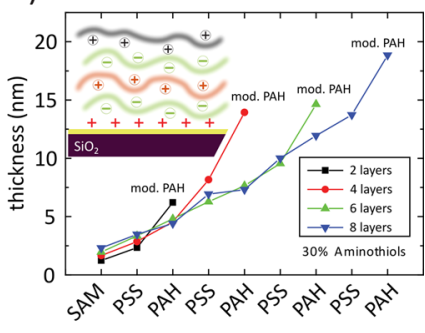

Figure 2. Multilayered polyelectrolytes and synthesis of thiolated poly allyl-amine (PAH). (a) Functionalization of $\mathrm{PAH}$ with a thiolated moiety. (b) Solid-state C-13 NMR of PAH (brown) and thiolated PAH (black). The methyl peak shifts from 32.98 to $36.78 \mathrm{ppm}$ following the conversion of the primary amine into a secondary amine linked to two methyl groups by a carbonyl functionality, responsible for the peak at $172.98 \mathrm{ppm}$. (c) Characterization of the sequential adsorption of the multilayered films of polyelectrolytes by ellipsometry.

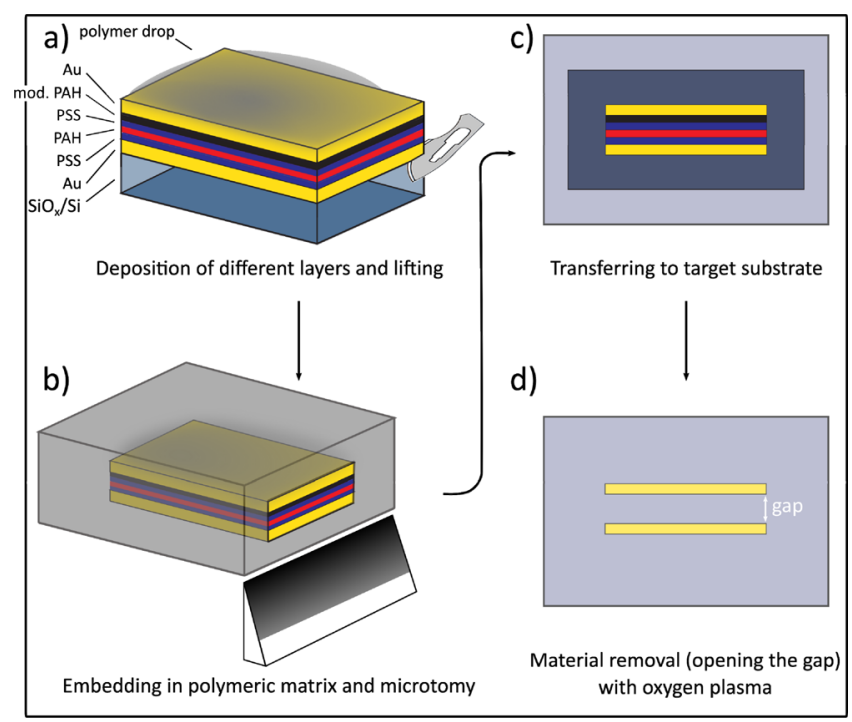

Figure 3. Process flow for the fabrication of metallic nanogaps: (a) The multilayered film of polyelectrolytes and gold is prepared on a $\mathrm{Si} /$ $\mathrm{SiO}_{2}$ wafer. The stack is lifted from the substrate and (b) embedded inside a polymer matrix. Microtomy of the polymer block yields a multilayered structure in a polymeric slab. (c) The slab is deposited onto an arbitrary substrate (top view representation) and (d) the gap is formed via oxygen plasma reactive etching of both the polymer support and the sacrificial layer of polyelectrolytes. The schematics are not on scale.

zine-2,4,6-trione, ${ }^{40-42}$ yielding a polymer block embedding the gold layers separated by the molecular spacer (Figure $3 b$ ). A diamond knife then slices the polymer block via microtomy, forming polymeric slabs embedding two nanorods spaced by the multilayered polyelectrolyte template. The thickness of the slabs is precisely controllable in a wide range (tens of nanometers to micrometers). The polymer support allows the facile transfer of the slabs from the diamond knife to a given substrate, here a silicon wafer (Figure 3c). Then, as a last step, a reactive oxygen plasma is used to etch the sacrificial film of polyelectrolytes and (for longer exposures) the supporting polymer, yielding a planar nanogap/nanochannel across metallic walls (Figure $3 d)$.

The pressure exerted on the block by the microtomy blade is potentially capable of compressing and narrowing the polyelectrolyte multilayer. The polymer, however, surrounds the polyelectrolyte structure entirely and absorbs/damps a large part of the stress. In practice, while the length of the nanogaps/nanochannels is limited to few tens of microns, the width of the polymeric slabs can be as large as few millimeters (the schematics in Figure 3 are not on scale) highlighting the importance of this damping effect by the polymer. Separately, the direction of the cutting in microtomy plays an important role in avoiding delamination and imperfections: proceeding with "vertical cut", with the blade orthogonal to the embedded multilayers generates an expanding stress across the multilayers, potentially capable to buckle the Au films, yielding the rupture of the structure by delamination. The compressive stress in a "horizontal cut" (blade/multilayer orientation shown in Figure 3b) however helps preventing the delamination of the assembly pushing the bottom Au film "up" and keeping the structure compressed. The sharpness of the diamond blade and the cutting speed are other important parameters. Slicing too slow $(0.2-0.5 \mathrm{~mm} / \mathrm{s})$ induces longstanding compressive pressures which ultimately causes delamination. Blunt blades exert friction onto the polymer, creating imperfections (e.g., delamination, deformation) especially at the interface between different layers.

The lateral size of the slab could vary in a large range, from tens of microns to even millimeters (the slabs shown in Figure 4a are of $~ 400$
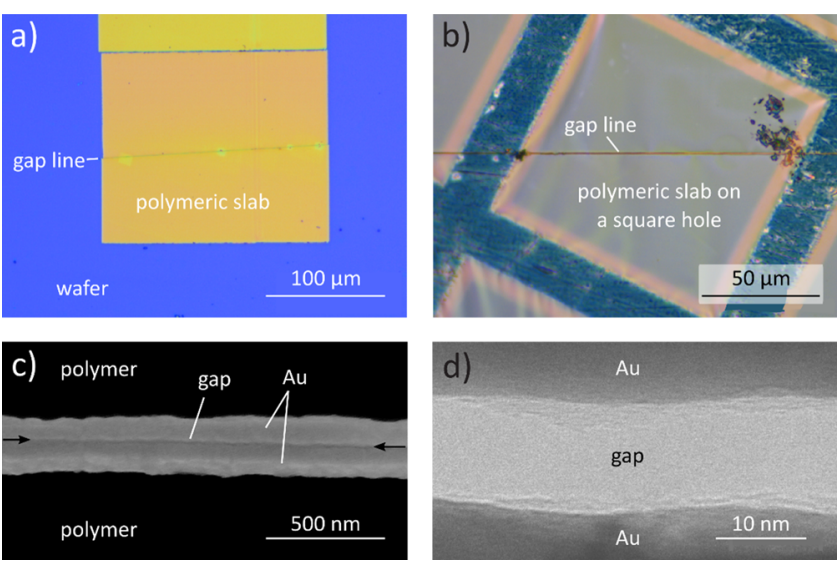

Figure 4. Characterization of the eventual nanogap/nanochannel structures. (a,b) Optical microscopy images demonstrating polymeric slabs containing multilayered polyelectrolytes/gold structure (gap line) and deposited on a $\mathrm{Si} / \mathrm{SiO}_{2}$ wafer (a) and a holey carbon grid (b). (c) Scanning electron microscopy (SEM) imageof a nanogaps/ nanochannels. (d) Transmission electron microscopy (TEM) image of a nanogap/nanochannel formed by four layers of polyelectrolytes. All the images are top-view oriented.

$\left.\times 400 \mu \mathrm{m}^{2}\right)$. As for the thickness, the microtomy can cut slices as thin as $\sim 50 \mathrm{~nm}$ effortlessly and can be as thick as several micrometers. Beyond the scalable preparation of the slabs, microtomy yields nanoarchitectures that can be easily manipulated, thanks to the surface tension between the water and the floating slabs embedding the eventual devices. Importantly, the supporting polymer and the sacrificial film of polyelectrolytes promote the integrity of the layered structure that can therefore be readily transferred onto arbitrary substrates, such as $\mathrm{Si} / \mathrm{SiO}_{2}$ wafers, transmission electron microscopy (TEM) grids, or SiN membranes (Figure $4 \mathrm{a}, \mathrm{b}$ ). In fact, after the microtomy step, the slabs slide from the diamond knife and float on a 
water meniscus placed behind the blade. Next, by positioning a titanium ring around the floating polymeric slabs, every device can be handled and transferred at will from the meniscus to a substrate of interest. In fact, the ring exploits the surface tension of the water to withdraw a droplet containing the floating polymeric slabs, allowing the agile transfer of the droplet onto any target substrate. After the transfer of the droplet, and while the water slowly evaporates, the polymeric slab steadily lands over the target substrate. The slow evaporation of water also permits the micrometer-scale alignment of the slab over microfabricated carrier chips using a needle connected to a microstep manipulator pinning the polymer slab and controlling its position.

The resulting slab after transfer on a $\mathrm{Si} / \mathrm{SiO}_{2}$ wafer was imaged using a scanning electron microscope (Figure 4c) showing an in-plane nanogap/nanochannel structure. Transmission electron microscopy (TEM) resolved the spacing with nanometer accuracy for a device suspended over a holey carbon grid (Figure $4 \mathrm{~d}$ ). The darker regions on the TEM image correspond to the two gold rods separated by a gap (brighter region) opened after the etching of a four-layer polyelectrolyte stack; the estimated gap width $(\sim 13 \mathrm{~nm})$ matches the ellipsometry results shown in Figure $2 c$.

\section{CONCLUSIONS}

To conclude, multilayered films of polyelectrolytes were deposited LbL on gold substrates and converted into nanogap/nanochannel structures via microtomy. The LbL deposition allowed the facile assembly of nanometric thin films over large area substrates, with thicknesses that could be readily monitored by optical methods such as ellipsometry. The further mechanical sectioning of such assemblies yielded spacings between two gold layers directly embedded within polymeric supports which were precisely transferred onto target substrates. Specifically, transferring a droplet of water with a floating integrated nanogap/nanochannel architecture in combination with proper alignment tools (i.e., a simple micromanipulator) allowed the precise control over the position on the target substrate. Our fabrication approach based on supramolecular templating will pave the way toward the design of a large variety of nanogaps/nanochannels where the spacing is precisely customized by structures as simple as macromolecular assemblies.

\section{ASSOCIATED CONTENT}

\section{SI Supporting Information}

The Supporting Information is available free of charge at https://pubs.acs.org/doi/10.1021/acsanm.0c01578.

Fabrication of the SAMs, LbL deposition, processing of the embedding polymer, microtomy of the metallic nanogaps, synthesis of the thiolated polyelectrolytes and IR characterization of the thiolated $\mathrm{PAH}$, and scalability and reproducibility of the structures (PDF)

\section{AUTHOR INFORMATION}

\section{Corresponding Author}

Grégory F. Schneider - Faculty of Science, Leiden Institute of Chemistry, Leiden University, 2333CC Leiden, The Netherlands; 이이.org/0000-0001-5018-3309; Email: g.f.schneider@chem.leidenuniv.nl

\section{Authors}

Hadi Arjmandi-Tash - Faculty of Science, Leiden Institute of Chemistry, Leiden University, 2333CC Leiden, The

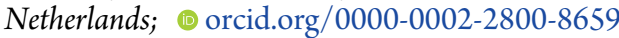

Pauline M.G. van Deursen - Faculty of Science, Leiden Institute of Chemistry, Leiden University, 2333CC Leiden, The Netherlands

Amedeo Bellunato - Faculty of Science, Leiden Institute of Chemistry, Leiden University, 2333CC Leiden, The Netherlands; (1) orcid.org/0000-0001-7016-4738

Clarisse de Sere - Faculty of Science, Leiden Institute of Chemistry, Leiden University, 2333CC Leiden, The Netherlands; (1) orcid.org/0000-0003-1781-5858

Zhanna Overchenko - Faculty of Science, Leiden Institute of Chemistry, Leiden University, 2333CC Leiden, The Netherlands; 이이이.org/0000-0001-8688-3681

Karthick Babu Sai Sankar Gupta - Faculty of Science, Leiden Institute of Chemistry, Leiden University, 2333CC Leiden, The Netherlands; (1) orcid.org/0000-0002-3528-2912

Complete contact information is available at:

https://pubs.acs.org/10.1021/acsanm.0c01578

\section{Author Contributions}

${ }^{\dagger}$ H.A.-T., P.M.G.v.D. and A.B. contributed equally to this work. Notes

The authors declare no competing financial interest.

\section{ACKNOWLEDGMENTS}

We would like to acknowledge the European Research Council under the European Union's Seventh Framework Program (FP/2007-2013)/ERC Grant agreement no. 335879 project acronym "Biographene". We also would like to acknowledge Prof. dr. Bram Koster and Eric Bos of the Leiden University Medical Centrum for providing assistance in the use of their LEICA ultramicrotome. We would also like to acknowledge Cristian van Helvoirt for the XPS analysis.

\section{REFERENCES}

(1) Dubois, V.; Bleiker, S. J.; Stemme, G.; Niklaus, F. Scalable Manufacturing of Nanogaps. Adv. Mater. 2018, 30, 1801124.

(2) Yang, Y.; Gu, C.; Li, J. Sub-5 Nm Metal Nanogaps: Physical Properties, Fabrication Methods, and Device Applications. Small 2019, 15, 1804177.

(3) Reed, M. A. Conductance of a Molecular Junction. Science 1997, $278,252-254$.

(4) Huang, S.; He, J.; Chang, S.; Zhang, P.; Liang, F.; Li, S.; Tuchband, M.; Fuhrmann, A.; Ros, R.; Lindsay, S. Identifying Single Bases in a DNA Oligomer with Electron Tunnelling. Nat. Nanotechnol. 2010, 5, 868-873.

(5) Liang, X.; Chou, S. Y. Nanogap Detector Inside Nanofluidic Channel for Fast Real-Time Label-Free DNA Analysis. Nano Lett. 2008, 8, 1472-1476.

(6) Fanget, A.; Traversi, F.; Khlybov, S.; Granjon, P.; Magrez, A.; Forró, L.; Radenovic, A. Nanopore Integrated Nanogaps for DNA Detection. Nano Lett. 2014, 14, 244-249.

(7) Li, T.; Hu, W.; Zhu, D. Nanogap Electrodes. Adv. Mater. 2010, $22,286-300$

(8) Hwang, J. S.; Kong, K. J.; Ahn, D.; Lee, G. S.; Ahn, D. J.; Hwang, S. W. Electrical Transport through 60 Base Pairs of Poly(DG)Poly(DC) DNA Molecules. Appl. Phys. Lett. 2002, 81, 1134-1136.

(9) Kyu Kim, S.; Cho, H.; Park, H.-J.; Kwon, D.; Min Lee, J.; Hyun Chung, B. Nanogap Biosensors for Electrical and Label-Free Detection of Biomolecular Interactions. Nanotechnology 2009, 20, 455502.

(10) Porath, D.; Bezryadin, A.; de Vries, S.; Dekker, C. Direct Measurement of Electrical Transport through DNA Molecules. Nature 2000, 403, 635-638. 
(11) Ward, D. R.; Hüser, F.; Pauly, F.; Cuevas, J. C.; Natelson, D. Optical Rectification and Field Enhancement in a Plasmonic Nanogap. Nat. Nanotechnol. 2010, 5, 732-736.

(12) Wu, H.-Y.; Choi, C. J.; Cunningham, B. T. Plasmonic NanogapEnhanced Raman Scattering Using a Resonant Nanodome Array. Small 2012, 8, 2878-2885.

(13) Chen, X.; Ciracì, C.; Smith, D. R.; Oh, S.-H. NanogapEnhanced Infrared Spectroscopy with Template-Stripped Wafer-Scale Arrays of Buried Plasmonic Cavities. Nano Lett. 2015, 15, 107-113.

(14) Marqués-González, S.; Matsushita, R.; Kiguchi, M. Surface Enhanced Raman Scattering of Molecules in Metallic Nanogaps. J. Optic. 2015, 17, 114001.

(15) Siegfried, T.; Ekinci, Y.; Martin, O. J. F.; Sigg, H. Gap Plasmons and Near-Field Enhancement in Closely Packed Sub-10 Nm Gap Resonators. Nano Lett. 2013, 13, 5449-5453.

(16) Chen, X.; Park, H.-R.; Pelton, M.; Piao, X.; Lindquist, N. C.; Im, H.; Kim, Y. J.; Ahn, J. S.; Ahn, K. J.; Park, N.; et al. Atomic Layer Lithography of Wafer-Scale Nanogap Arrays for Extreme Confinement of Electromagnetic Waves. Nat. Commun. 2013, 4, 2361.

(17) Lindquist, N. C.; Nagpal, P.; McPeak, K. M.; Norris, D. J.; Oh, S.-H. Engineering Metallic Nanostructures for Plasmonics and Nanophotonics. Rep. Prog. Phys. 2012, 75, 036501.

(18) Wiley, B. J.; Lipomi, D. J.; Bao, J.; Capasso, F.; Whitesides, G. M. Fabrication of Surface Plasmon Resonators by Nanoskiving SingleCrystalline Gold Microplates. Nano Lett. 2008, 8, 3023-3028.

(19) Arjmandi-Tash, H.; Bellunato, A.; Wen, C.; Olsthoorn, R. C.; Scheicher, R. H.; Zhang, S.-L.; Schneider, G. F. Zero-Depth Interfacial Nanopore Capillaries. Adv. Mater. 2018, 30, 1703602.

(20) Perrin, M. L.; Burzurí, E.; Van Der Zant, H. S. J. J. Chem Soc Rev Single-Molecule Transistors. Chem. Soc. Rev. 2015, 44, 902-919.

(21) Tsutsui, M.; Taniguchi, M.; Yokota, K.; Kawai, T. Identifying Single Nucleotides by Tunnelling Current. Nat. Nanotechnol. 2010, 5, 286-290.

(22) Perrin, M. L.; Verzijl, C. J. O.; Martin, C. A.; Shaikh, A. J.; Eelkema, R.; Van Esch, J. H.; Van Ruitenbeek, J. M.; Thijssen, J. M.; Van Der Zant, H. S. J.; Dulić, D. Large Tunable Image-Charge Effects in Single-Molecule Junctions. Nat. Nanotechnol. 2013, 8, 282-287.

(23) Martin, C. A.; Smit, R. H. M.; van der Zant, H. S. J.; van Ruitenbeek, J. M. A Nanoelectromechanical Single-Atom Switch. Nano Lett. 2009, 9, 2940-2945.

(24) Martin, C. A.; Ding, D.; Van Der Zant, H. S. J.; Ruitenbeek, J. M. v. Lithographic Mechanical Break Junctions for Single-Molecule Measurements in Vacuum: Possibilities and Limitations. New J. Phys. 2008, 10, 065008.

(25) Martin, C. A.; Ding, D.; Sørensen, J. K.; Bjørnholm, T.; van Ruitenbeek, J. M.; van der Zant, H. S. J. J. Fullerene-Based Anchoring Groups for Molecular Electronics. J. Am. Chem. Soc. 2008, 130, 13198-13199.

(26) McCord, M. A.; Rooks, M. J. Electron Beam Lithography. In Handbook of Microlithography, Micromachining, and Microfabrication: Microlithography; Rai-Choudhury, P., Ed.; SPIE PRESS, 1997; Volume 1, pp 139-250.

(27) Lipomi, D. J.; Martinez, R. V.; Whitesides, G. M. Use of Thin Sectioning (Nanoskiving) to Fabricate Nanostructures for Electronic and Optical Applications. Angew. Chem., Int. Ed. 2011, 50, 85668583.

(28) Blees, M. K.; Barnard, A. W.; Rose, P. a.; Roberts, S. P.; McGill, K. L.; Huang, P. Y.; Ruyack, A. R.; Kevek, J. W.; Kobrin, B.; Muller, D. a.; et al. Graphene Kirigami. Nature 2015, 524, 204-207.

(29) Handbook of Thin Film Deposition; Seshan, K., Schepis, D., Eds.; Elsevier, 2018.

(30) Park, H.; Lim, A. K. L.; Alivisatos, A. P.; Park, J.; McEuen, P. L. Fabrication of Metallic Electrodes with Nanometer Separation by Electromigration. Appl. Phys. Lett. 1999, 75, 301-303.

(31) Lambert, M. F.; Goffman, M. F.; Bourgoin, J. P.; Hesto, P. Fabrication and Characterization of Sub-3 Nm Gaps for SingleCluster and Single-Molecule Experiments. Nanotechnology 2003, 14, $772-777$.
(32) Ji, D.; Li, T.; Fuchs, H. Nanosphere Lithography for Sub-10$\mathrm{Nm}$ Nanogap Electrodes. Adv. Mater. Optic. Electron. 2017, 3, 1600348.

(33) Johnston, D. E.; Strachan, D. R.; Johnson, A. T. C. Parallel Fabrication of Nanogap Electrodes. Nano Lett. 2007, 7, 2774.

(34) Esen, G.; Fuhrer, M. S. Temperature Control of Electromigration to Form Gold Nanogap Junctions. Appl. Phys. Lett. 2005, 87,263101

(35) T., Taychatanapat; K. I., Bolotin; F., Kuemmeth; Ralph, D. C. Imaging Electromigration during the Formation of Break Junctions. 2007, 7. 652. https://doi.org/ DOI: 10.1021/NL062631I.

(36) Decher, G. Fuzzy Nanoassemblies: Toward Layered Polymeric Multicomposites. Science 1997, 277, 1232-1237.

(37) Storm, A. J.; Chen, J. H.; Ling, X. S.; Zandbergen, H. W.; Dekker, C. Fabrication of Solid-State Nanopores with SingleNanometre Precision. Nat. Mater. 2003, 2, 537-540.

(38) Carlotti, M.; Degen, M.; Zhang, Y.; Chiechi, R. C. Pronounced Environmental Effects on Injection Currents in EGaIn Tunneling Junctions Comprising Self-Assembled Monolayers. J. Phys. Chem. C 2016, 120, 20437-20445.

(39) Love, J. C.; Estroff, L. A.; Kriebel, J. K.; Nuzzo, R. G.; Whitesides, G. M. Self-Assembled Monolayers of Thiolates on Metals as a Form of Nanotechnology. Chem. Rev. 2005, 105, 1103-1170.

(40) Mays, R. L.; Pourhossein, P.; Savithri, D.; Genzer, J.; Chiechi, R. C.; Dickey, M. D. Thiol-Containing Polymeric Embedding Materials for Nanoskiving. J. Mater. Chem. C 2013, 1, 121-130.

(41) Lu, H.; Carioscia, J. A.; Stansbury, J. W.; Bowman, C. N. Investigations of Step-Growth Thiol-Ene Polymerizations for Novel Dental Restoratives. Dent. Mater. 2005, 21, 1129-1136.

(42) Carioscia, J. A.; Stansbury, J. W.; Bowman, C. N. Evaluation and Control of Thiol-Ene/Thiol-Epoxy Hybrid Networks. Polymer 2007, 48, 1526-1532. 